\title{
ECOLOGIA DE COMUNIDADES DE INSETOS BENTÔNICOS (EPHEMEROPTERA, PLECOPTERA E TRICHOPTERA), EM CÓRREGOS DO PARQUE ECOLÓGICO DE GOIÂNIA, GOIÁS, BRASIL
}

\author{
Leandro Gonçalves Oliveira ${ }^{1}$ \\ Pitágoras da Conceição Bispo ${ }^{2}$ \\ Nívia Custódio de Sá ${ }^{1}$
}

\begin{abstract}
ECOLOGY OF BENTHIC INSECTS COMMUNITIES (EPHEMEROPTERA, PleCOPtera and Trichoptera) in streams at Parque Ecológlco de Goiñnia, Goińs, BRAZIL. Relationships among some abiotic factors (temperature, velocity, flow, conductivity, oxy-reduction potential and $\mathrm{pH}$ ) and the seazonal density of benthic insects (immature forms) of the orders Ephemeroptera, Plecoptera and Trichoptera at the Parque Ecológico de Goiânia, Goiás are studied. An area of $1 \mathrm{~m}^{2}$ was sampled and abiotic factors were recorded in 4 sites from August/1994 to July/1995. The faunistic composition was $75 \%$ Ephemeroptera, $1 \%$ Plecoptera and $24 \%$ Trichoptera. The data suggest the water velocity and flow as the main factors that determined the aquatic insects abundance.

KEY WORDS. Community ecology, aquatic insects, lotic, abiotic factors
\end{abstract}

Os estudos sobre a história natural de insetos aquáticos de ambientes lóticos brasileiros, principalmente aqueles de aspectos mais gerais sobre a composição faunística (SCHROEDER-ARAUJO et al. 1986; FERREIRA \& FroEHLICH 1992; OLIVEIRA \& FROEHLICH 1996), taxonomia (FROEHLICH 1984a,b, 1988, 1990a, 1991, 1994, 1996; GUAHYBA 1988), morfometria (FROEHLICH 1990b) e biomonitoramento (SCHROEDER-ARAUJO \& CIPOLLI 1986), tiveram um pequeno incremento a partir do trabalho de VANZOLINI (1964), que serviu para constatar a situação incipiente do conhecimento sobre diversos organismos aquáticos no Brasil.

Na região do Brasil Central, mais especificamente em córregos de Goiás, alguns estudos foram realizados sobre a estrutura de comunidades de insetos aquáticos e padrões de distribuição espacial (BISPO \& OLIVEIRA no prelo), principalmente para as formas imaturas das ordens Ephemeroptera, Plecoptera e Trichoptera $(\mathrm{EPT})$.

1) Departamento de Biologia Geral, Instituto de Ciências Biológicas, Universidade Federal de Goiás. Caixa Postal 131, 74001-970 Goiânia, Goiás, Brasil.

2) Faculdade de Filosofia, Ciências e Letras de Ribeirão Preto, Universidade de São Paulo. Avenida Bandeirantes, 4800, 14040-901 Ribeirão Preto, São Paulo, Brasil. Bolsista da CAPES. 
Diante do exposto, o presente trabalho tem como objetivo a realização de um levantamento da composição faunística dos referidos táxons na região do Parque Ecológico de Goiânia, principalmente por se tratar de um local com relativa proteção ambiental, bem como a relação entre a distribuição temporal das larvas e ninfas e alguns fatores abióticos pertinentes.

\section{MATERIAL E MÉTODOS}

\section{Área de Estudo}

O estudo foi conduzido no Córrego do Macacos, Córrego Carapina e Ribeirão João Leite, na região do Parque Ecológico de Goiânia, Goiás (Fig. 1). O Ribeirão João Leite tem seu leito parcialmente sombreado pela vegetação marginal no trecho dentro dos limites do parque. Possui grande volume de água e é profundo em vários locais $(>1,5 \mathrm{~m})$, apresentando fundo pedregoso somente nos trechos de maior velocidade. O Córrego Carapina está localizado dentro de uma mata relativamente densa, porém possui alguns trechos pouco sombreados. O córrego apresenta fundo pedregoso e profundidade reduzida $(<0,5 \mathrm{~m})$. O Córrego dos Macacos é o único afluente do Córrego Carapina, possuindo um leito protegido por uma vegetação densa; entretanto, a sua nascente está localizada fora dos limites do parque, sendo bastante devastada e com pouca vegetação.

\section{Coletas}

Foram realizadas coletas mensais no período de agosto/94 a julho/95, em quatro pontos: Ponto 1) Córrego dos Macacos (primeira ordem); Ponto 2) Córrego Carapina (trecho de primeira ordem); Ponto 3) Córrego Carapina (trecho de segunda ordem); e Ponto 4) Ribeirão João Leite (quarta ordem). Essa classificação hidrológica foi baseada em STRAHLER (1957). As coletas foram realizadas em regiões de corredeira e fundo pedregoso, com auxílio de um amostrador de Surber (MERRITT \& CUMMINS 1979), totalizando $1 \mathrm{~m}^{2}$ de área amostrada por ponto. Os organismos previamente triados foram fixados em formol $5 \%$, identificados e quantificados, sendo posteriormente acondicionados em álcool $80 \%$.

Os estágios imaturos foram identificados em nível de família, utilizando-se as chaves taxonômicas de DOMINGUEZ et al. (1992) para efemerópteros; BACHMANN (1995) para plecópteros e AGRISANO (1995) para os tricópteros.

Alguns fatores abióticos, como temperatura do ar e da água, velocidade da água, vazão, condutividade elétrica, potencial de oxi-redução, potencial hidrogeniônico $(\mathrm{pH})$ e precipitação pluviométrica, foram registrados para os pontos de coleta. As temperaturas da água e do ar foram obtidas com auxílio de um termômetro a álcool $\left(0-50^{\circ} \mathrm{C}\right)$. A velocidade da água foi mensurada através do método do flutuador e a vazão calculada através do produto da velocidade média da água por uma área de secção feita no córrego (LiND 1979). A condutividade elétrica da água, o potencial de oxi-redução e o pH foram obtidos através de um condutivímetro de campo Corning PS-17, um oxímetro de campo Corning PS-19 e um pHmetro de campo Corning PS-15, respectivamente. O oxigênio dissolvido não foi registrado. Os valores da precipitação média mensal foram obtidos junto à Escola de Agrono- 
mia da Universidade Federal de Goiás, através de sua estação evaporimétrica, com coordenardas geográficas de $16^{\circ} 41^{\prime} \mathrm{S}$ e $49^{\circ} 17^{\prime} \mathrm{W}$.

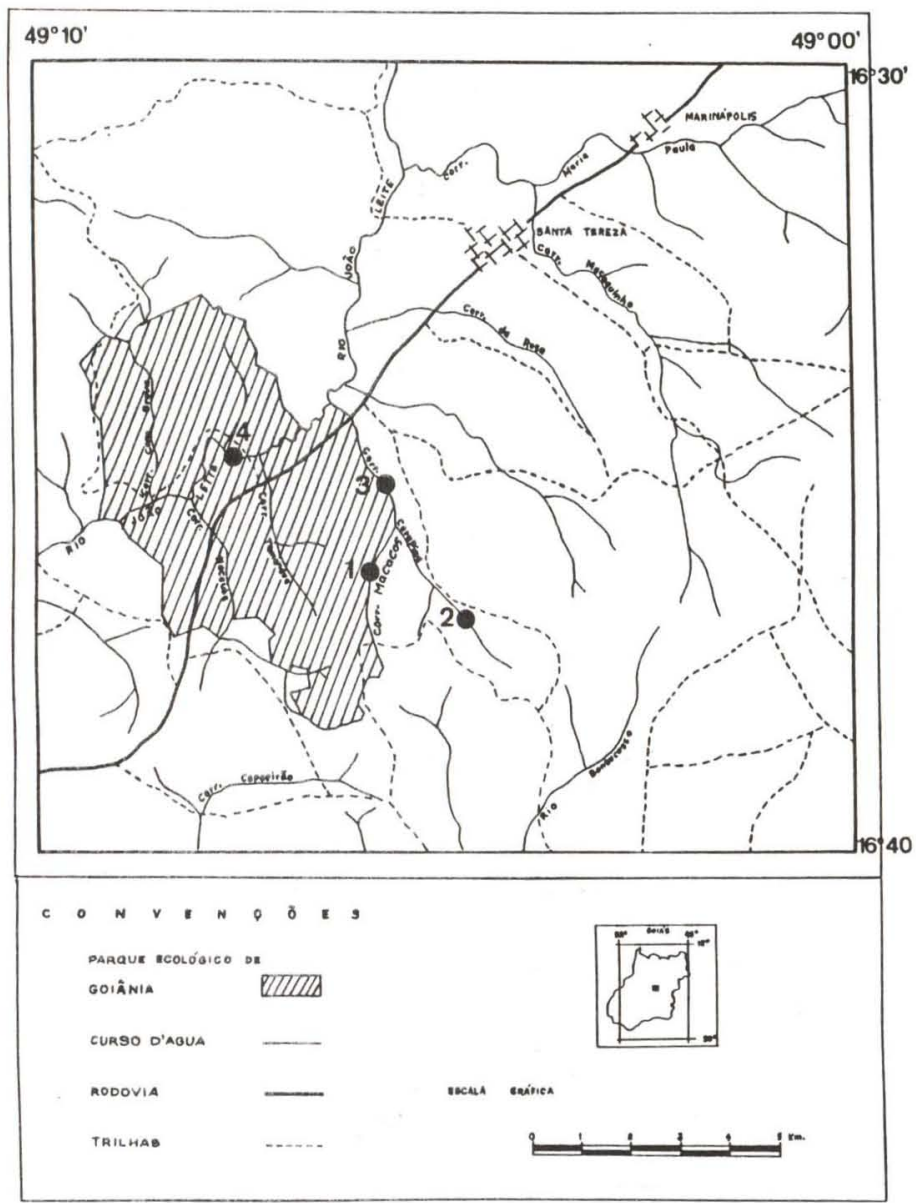

Fig. 1. Mapa do Parque Ecológico de Goiânia, Goiás, indicando os pontos de coleta: (1) Córrego dos Macacos primeira ordem; (2) Córrego Carapina primeira ordem; (3) Córrego Carapina segunda ordem; e (4) Ribeirão João Leite quarta ordem; dos estágios imaturos de Ephemeroptera, Plecoptera e Trichoptera.

\section{Análise Estatística}

A diversidade e a equidistribuição dos organismos foram calculadas em nível taxonômico de família, para cada ponto em todas as coletas, utilizando o Índice de Shannon-Wiener e de Uniformidade (MAGURRAN 1991), respectivamente. O número total de EPT colecionados mensalmente foram correlacionados com a precipitação do mês anterior e com a do mês correspondente a coleta, através do índice de correlação de Pearson. 


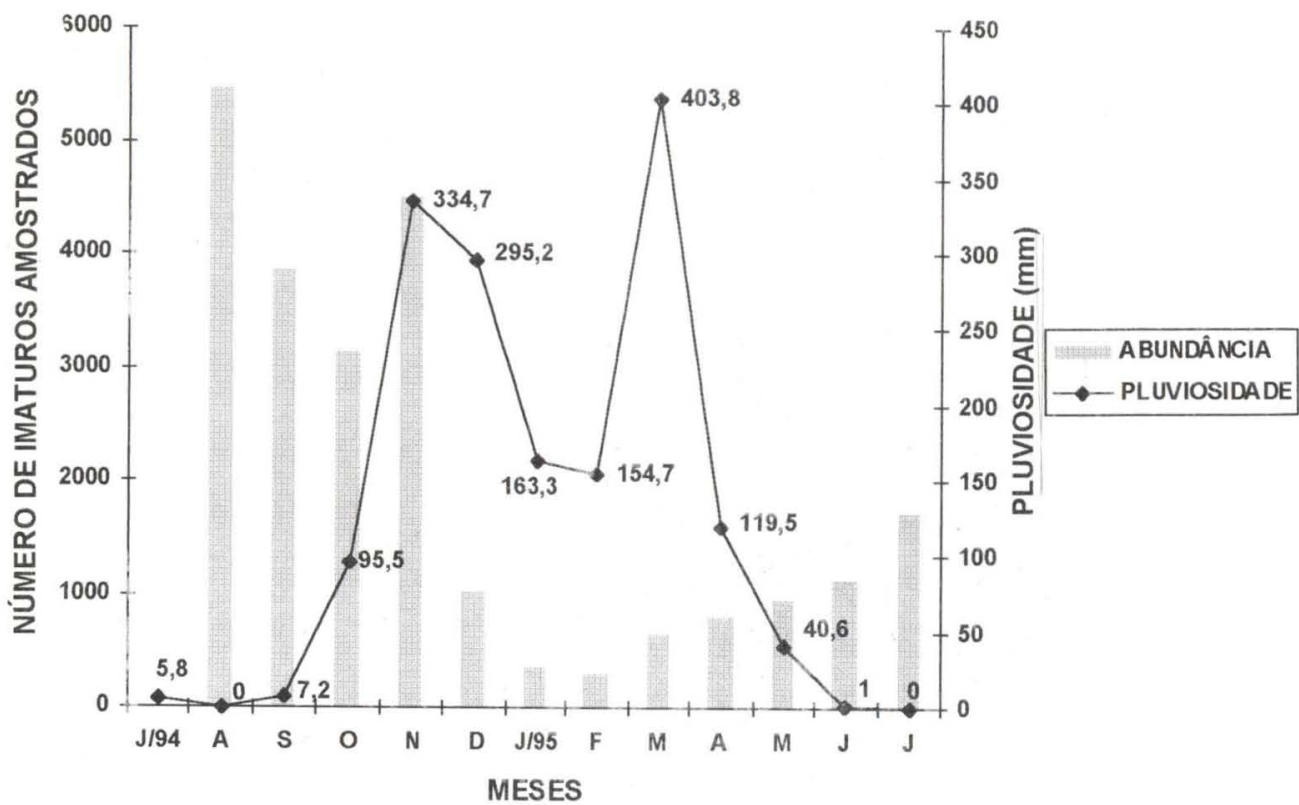

Fig. 2. Número total de imaturos das ordens Ephemeroptera, Plecoptera e Trichoptera, colecionados no periodo de agosto/1994 a julho/1995, e os valores da precipitação média registrados mensalmente na região do Parque Ecológico de Goiânia, Goiás, de julho/1994 a julho/1995.

\section{RESULTADOS E DISCUSSÃO}

A distribuição temporal e espacial dos insetos aquáticos em ambientes lóticos dependem de inúmeros fatores. Segundo SlobodChiKoff \& ParRotT (1977), nesses ambientes, a correnteza da água parece ser um dos que mais interferem na abundância das comunidades bentônicas. A temperatura, sobretudo em regiões de clima temperado, influencia tanto na ecologia quanto na evolução dos insetos aquáticos (WARD \& STANFORD 1982). Fatores como alimentação e condições físico-químicos da água ( $\mathrm{pH}$, oxigênio e condutividade) também influenciam na estruturação dessas comunidades (PETERSON \& EECKHAUTE 1992; ZAMORA-MUÑOZ et al. 1993).

Segundo NIMER (1989), a região estudada apresenta um clima tropical semi-úmido, com estação chuvosa no verão (grande pluviosidade entre dezembro e fevereiro) e uma estação seca no inverno (de maio a setembro). Os dados de pluviosidade do presente trabalho corroboram este padrão (Fig. 2).

BooN et al. (1986) colocam a pluviosidade e subseqüente inundação, como um fator que interfere de maneira muito significativa na abundância dos invertebrados bentônicos. No presente trabalho, observou-se um aumento da vazão no período chuvoso, porém os meses com maior precipitação não foram necessariamente aqueles de maior vazão. Isto provavelmente se deve ao fato de que os valores da vazão refletem o dia de coleta e não a vazão geral do mês, como os valores de 
precipitação registrados nesse trabalho. Esse fenômeno pode ser vizualizado mais claramente no ponto 4 , no mês de outubro, onde foi verificada a maior vazão, sendo que a precipitação mensal ainda era de $95,5 \mathrm{~mm}$.

Tabela I. Valores da Condutividade (Cond.), Potencial de Oxi-Redução (Orp.), Potencial Hidrogeniônico ( $\mathrm{pH}$ ), Temperatura da água (Tag.), Temperatura do ar (Tar), Velocidade (Vel.) e Vazão (Vaz.) da água registrados nos quatro pontos de amostragem, durante as coletas nos corpos aquáticos do Parque Ecológico de Goiânia, Goiás.

\begin{tabular}{|c|c|c|c|c|c|c|c|c|c|c|c|c|}
\hline & Ago/94 & Set & Out & Nov & Dez & Jan/95 & Fev & Mar & $\mathrm{Abr}$ & Mai & Jun & Jul \\
\hline \multicolumn{13}{|l|}{ Ponto 1} \\
\hline Cond $(\mu \mathrm{S} / \mathrm{cm})$ & 60,00 & 80,00 & 80,00 & 80,00 & 130,00 & 110,00 & 120,00 & 110,00 & 110,00 & 110,00 & 100,00 & 110,00 \\
\hline Orp (mV) & 118,00 & 113,00 & 103,00 & 180,00 & 117,00 & 170,00 & 61,00 & 35,00 & 71,00 & 52,00 & 33,00 & 120,00 \\
\hline $\mathrm{pH}$ & 7,00 & 6,80 & 6,90 & 7,00 & 7,20 & 6,80 & 7,00 & 7,00 & 6,90 & 6,80 & 6,90 & 6,80 \\
\hline Tag. $\left({ }^{\circ} \mathrm{C}\right)$ & 18,00 & 20,00 & 20,50 & 19,60 & 19,50 & 19,00 & 19,50 & 19,50 & 19,00 & 17,50 & 15,00 & 16,00 \\
\hline $\operatorname{Tar}\left({ }^{\circ} \mathrm{C}\right)$ & 18,00 & 22,00 & 24,00 & 21,00 & 19,50 & 23,50 & 20,50 & 21,50 & 20,50 & 29,50 & 17,00 & 19,00 \\
\hline Vel. $(\mathrm{m} / \mathrm{s})$ & 0,22 & 0,18 & 0,22 & 0,18 & 0,25 & 0,16 & 0,40 & 0,29 & 0,24 & 0,34 & 0,22 & 0,24 \\
\hline $\operatorname{Vaz}\left(\mathrm{m}^{3} / \mathrm{s}\right)$ & 0,02 & 0,01 & 0,01 & 0,01 & 0,52 & 1,09 & 0,48 & 0,36 & 0,73 & 0,50 & 0,70 & 0,33 \\
\hline \multicolumn{13}{|l|}{ Ponto 2} \\
\hline Cond $(\mu \mathrm{S} / \mathrm{cm})$ & 70,00 & 80,00 & 100,00 & 90,00 & 180,00 & 150,00 & 120,00 & 120,00 & 130,00 & 120,00 & 110,00 & 120,00 \\
\hline Orp (mV) & 181,00 & 173,00 & 157,00 & 188,00 & 129,00 & 172,00 & 127,00 & 142,00 & 128,00 & 115,00 & 103,00 & 150,00 \\
\hline $\mathrm{pH}$ & 7,90 & 7,70 & 7,50 & 7,70 & 7,70 & 7,30 & 7,20 & 1,10 & 7,20 & 7,30 & 7,30 & 6,90 \\
\hline Tag. $\left({ }^{\circ} \mathrm{C}\right)$ & 17,50 & 21,00 & 21,00 & 21,00 & 21,00 & 20,00 & 20,00 & 20,00 & 19,50 & 17,50 & 15,00 & 16,50 \\
\hline $\operatorname{Tar}\left({ }^{\circ} \mathrm{C}\right)$ & 22,00 & 28,00 & 25,00 & 18,00 & 19,00 & 26,00 & 20,00 & 21,00 & 22,00 & 20,00 & 17,00 & 22,00 \\
\hline Vel. $(\mathrm{m} / \mathrm{s})$ & 0,35 & 0,26 & 0,28 & 0,30 & 1,09 & 0,37 & 0,47 & 0,40 & 0,39 & 0,48 & 0,41 & 0,39 \\
\hline Vaz. $\left(\mathrm{m}^{3} / \mathrm{s}\right)$ & 0,21 & 0,11 & 0,14 & 0,12 & 0,53 & 1,38 & 1,65 & 1,48 & 1,73 & 1,18 & 1,47 & 1,87 \\
\hline \multicolumn{13}{|l|}{ Ponto 3} \\
\hline Cond $(\mu \mathrm{S} / \mathrm{cm})$ & 70,00 & 70,00 & 100,00 & 100,00 & 150,00 & 140,00 & 120,00 & 120,00 & 130,00 & 120,00 & 120,00 & 120,00 \\
\hline $\operatorname{Orp}(\mathrm{mV})$ & 200,00 & 209,00 & 157,00 & 148,00 & 148,00 & 158,00 & 120,00 & 97,00 & 126,00 & 100,00 & 75,00 & 123,00 \\
\hline $\mathrm{pH}$ & 7,60 & 7,70 & 7,70 & 7,80 & 7,70 & 7,80 & 7,50 & 7,60 & 7,50 & 7,50 & 7,60 & 7,30 \\
\hline Tag. $\left({ }^{\circ} \mathrm{C}\right)$ & 16,00 & 19,00 & 20,00 & 20,00 & 20,00 & 19,00 & 19.50 & 19,50 & 19,00 & 17,00 & 14,00 & 15,50 \\
\hline $\operatorname{Tar}\left({ }^{\circ} \mathrm{C}\right)$ & 20,5 & 27,00 & 20,00 & 23,00 & 20,50 & 20,00 & 19,00 & 20,00 & 20,00 & 18,00 & 15,00 & 19,20 \\
\hline Vel. $(\mathrm{m} / \mathrm{s})$ & 0,47 & 0,36 & 0,53 & 0,43 & 0,39 & 0,43 & 0,69 & 0,63 & 0,64 & 0,60 & 0,69 & 0,52 \\
\hline Vaz. $\left(\mathrm{m}^{3} / \mathrm{s}\right)$ & 0,14 & 0,08 & 0,11 & 0,07 & 0,64 & 0,86 & 0,82 & 0,76 & 0,68 & 0,74 & 0,34 & 0,81 \\
\hline \multicolumn{13}{|l|}{ Ponto 4} \\
\hline Cond $(\mu \mathrm{S} / \mathrm{cm})$ & 80,00 & 80,00 & 60,00 & 90,00 & 150,00 & 120,00 & 120,00 & 120,00 & 130,00 & 120,00 & 120,00 & 120,00 \\
\hline Orp (mV) & 150,00 & 161,00 & 112,00 & 168,00 & 106,00 & 164,00 & 160,00 & 122,00 & 134,00 & 127,00 & 120,00 & 154,00 \\
\hline $\mathrm{pH}$ & 7,00 & 7,80 & 6,90 & 7,60 & 7,60 & 7,10 & 7,10 & 7,20 & 7,10 & 7,40 & 7,30 & 7,40 \\
\hline Tag. $\left({ }^{\circ} \mathrm{C}\right)$ & 19,00 & 20,00 & 21,00 & 22,50 & 22,00 & 22,00 & 22,50 & 22,50 & 22,00 & 19,00 & 16,00 & 18,00 \\
\hline $\operatorname{Tar}\left({ }^{\circ} \mathrm{C}\right)$ & 23,50 & 27,00 & 19,00 & 21,50 & 20,50 & 25,00 & 21,00 & 24,50 & 21,00 & 20,00 & 25,00 & 23,00 \\
\hline Vel. $(\mathrm{m} / \mathrm{s})$ & 0,91 & 0,97 & 1,47 & 0,99 & 0,93 & 1.54 & 0,94 & 1.16 & 10,30 & 1.05 & 1,52 & 1,03 \\
\hline Vaz. $\left(\mathrm{m}^{3} / \mathrm{s}\right)$ & 5,94 & 9,30 & 23,20 & 6,40 & 10,10 & 10,50 & 14,10 & 9,70 & 8,53 & 10,36 & 5,85 & 8,42 \\
\hline
\end{tabular}

A pluviosidade, vazão e velocidade da água constituiram o conjunto de fatores que mais interferiram na densidade das formas imaturas de Ephemeroptera, Plecoptera e Trichoptera (EPT), como pode ser verificado examinando a figura 2 e as tabelas I e II. A correlação entre a pluviosidade do mês correspondente a coleta e a abundância não foi significativa $(\mathrm{r}=-0.216, \mathrm{p}>0,05)$, isto provavelmente pelo fato da pluviosidade corresponder a média mensal e a abundância ao dia de coleta, como já foi discutido anteriormente. Quando considerado os valores de pluviosidade do mês anterior, houve uma correlação negativa com o número de indivíduos colecionados $(r=-0,602, p<0,05)$, indicando que o efeito deste fator é dectado na fauna de EPT no mês subseqüente, devido a seu efeito acumulativo sobre a fauna. Essa correlação pode ser explicada pelo aumento do carreamento de sedimentos, de detritos vegetais e dos próprios organismos (drift), provocado pela elevação da 
velocidade e da vazão no período chuvoso. No período mais seco foram registradas as maiores abundâncias, sendo o valor máximo registrado em agosto, mês sem precitação. Esses dados corroboram o trabalho de BISPO \& OLIVEIRA (no prelo).

Tabela II. Número total de imaturos das ordens Ephemeroptera, Plecoptera e Trichoptera amostrados no Parque Ecológico de Goiânia, Goiás, no período de agosto/1994 à julho/1995, em quatro pontos de coleta. $\left(\mathrm{H}^{\prime}\right)$ Índice de Diversidade de Shannon-Wiener; (E) Índice de Uniformidade.

\begin{tabular}{|c|c|c|c|c|c|c|c|c|c|c|c|c|c|}
\hline & Ago/94 & Set & Out & Nov & Dez & $\operatorname{Jan} / 95$ & Fev & Mar & $\mathrm{Abr}$ & Mai & Jun & Jul & Total \\
\hline \multicolumn{14}{|l|}{ Ponto 1} \\
\hline laetidae & 145 & 253 & 324 & 165 & 27 & 9 & 4 & 12 & 10 & 81 & 33 & 113 & 1176 \\
\hline Caenidae & - & - & 2 & 1 & - & - & - & - & - & - & - & - & 2 \\
\hline Leptohyphidae & 197 & 211 & 518 & 523 & 97 & 31 & 45 & 34 & 14 & 44 & 30 & 60 & 1804 \\
\hline Leptophlebiidae & 15 & 31 & 111 & 178 & 43 & 15 & 14 & 18 & 28 & 31 & 38 & 31 & 553 \\
\hline Perlidae & 10 & 18 & 9 & 15 & 15 & 5 & 7 & 8 & 11 & 12 & 7 & 51 & 168 \\
\hline Calamoceratidae & - & 8 & 18 & 8 & - & - & - & - & - & - & - & - & 34 \\
\hline Glossosomatidae & - & 1 & - & - & - & - & - & - & - & - & 1 & - & 2 \\
\hline Helicopsychidae & - & - & 1 & - & - & - & - & - & - & - & - & - & 1 \\
\hline Hydrobiosidae & 8 & 16 & 11 & 20 & 2 & - & - & - & 2 & - & - & 7 & 66 \\
\hline Hydropsychidae & 76 & 46 & 132 & 179 & 58 & 28 & 11 & 15 & 20 & 67 & 28 & 10 & 670 \\
\hline Hydroptilidae & 7 & 16 & 10 & 26 & 3 & - & 11 & - & - & 1 & - & 2 & 76 \\
\hline Leptoceridae & - & - & 1 & - & 2 & - & - & - & 1 & - & - & - & 4 \\
\hline Odonto & 2 & 2 & - & - & 1 & - & - & - & - & - & - & - & $\mathbf{n}^{-}$ \\
\hline Philo & 2 & 10 & 36 & 24 & 2 & - & - & 1 & 1 & 5 & 4 & 3 & 88 \\
\hline Poly & - & - & 1 & - & 1 & - & - & 3 & - & - & 1 & - & 6 \\
\hline Xiphocentronidae & 8 & 16 & 11 & 20 & - & - & - & - & - & - & - & - & 55 \\
\hline Total & 470 & 628 & 1185 & 1159 & 251 & 88 & 92 & 91 & 87 & 241 & 142 & 277 & 4711 \\
\hline $\mathrm{H}^{\prime}$ & 3,459 & 3,585 & 3,807 & 3,459 & 3,459 & 2,322 & 2,585 & 2,807 & .000 & 3,000 & 3,000 & 3,000 & 2,426 \\
\hline$E$ & 0,622 & 0,646 & 0,586 & 674 & 0,679 & 0,888 & 0,824 & 0,837 & 0,816 & 0,762 & 0,798 & 0,736 & 0,606 \\
\hline \multicolumn{14}{|c|}{ 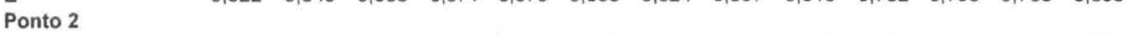 } \\
\hline Baetidae & 180 & 250 & 81 & 26 & 9 & 9 & 1 & 1 & 7 & 14 & 8 & - & 586 \\
\hline phidae & 653 & 171 & 752 & 1122 & 157 & 43 & 4 & 32 & 39 & 47 & 34 & 68 & 3122 \\
\hline Lept: & 26 & 26 & 5 & 6 & 1 & - & - & - & 2 & 4 & 2 & 126 & 198 \\
\hline Perl & 1 & - & - & - & 1 & - & - & - & - & - & - & - & 0 \\
\hline Cal & - & 1 & 3 & - & - & - & - & - & - & - & - & - & 4 \\
\hline $\mathrm{Hyc}$ & 1 & - & ( & - & - & - & - & - & - & - & - & - & 1 \\
\hline $\mathrm{Hy}$ & 73 & 59 & 108 & 109 & 40 & 23 & 13 & 35 & 46 & 57 & 38 & 71 & 672 \\
\hline $\mathrm{Hy}$ & 2 & - & 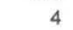 & 6 & 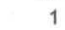 & 1 & - & - & - & - & - & - & 14 \\
\hline Lep & - & - & 1 & 2 & - & - & - & - & 1 & 1 & - & - & \\
\hline $\mathrm{Ph}$ & 239 & 135 & 21 & 19 & 17 & 4 & - & 6 & 38 & 71 & 51 & 65 & 666 \\
\hline ae & 1 & - & - & - & - & - & - & - & - & - & - & - & $=$ \\
\hline Total & 1176 & 642 & 975 & 1290 & 226 & 80 & 18 & 74 & 133 & 194 & 133 & 330 & 5271 \\
\hline $\mathrm{H}^{\prime}$ & 70 & 2,585 & 3,000 & 2,807 & 2,585 & 2,322 & 1,585 & 2,000 & 2,585 & 2,585 & 2,322 & 2,000 & 1,783 \\
\hline$E$ & 557 & 0,785 & 0,352 & 0,273 & 0,519 & 0,710 & 0,664 & 0,706 & 0,764 & 0,764 & 0,812 & 0,845 & 0,515 \\
\hline \multicolumn{14}{|l|}{ Ponto 3} \\
\hline Baetidae & 349 & 413 & 136 & 46 & 23 & 23 & 5 & 19 & 15 & 29 & 33 & 7 & 1098 \\
\hline $\mathrm{Ca}$ & - & - & 1 & - & - & - & - & - & - & - & - & - & 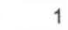 \\
\hline Lep & 1700 & 1011 & 681 & 675 & 244 & 66 & 67 & 93 & 131 & 149 & 128 & 173 & 5118 \\
\hline lebiidae & 185 & 19 & 3 & 12 & 1 & - & 1 & 9 & 17 & 25 & 33 & 4 & 309 \\
\hline Per & 3 & 4 & - & - & - & 1 & 1 & - & - & 6 & - & - & 15 \\
\hline Cal & - & 2 & 9 & - & - & - & - & 1 & - & - & - & - & 12 \\
\hline Glo: & 16 & 5 & 12 & 4 & 1 & - & - & - & 1 & 5 & 2 & 4 & 50 \\
\hline Helic & - & 1 & - & - & - & - & - & - & - & - & - & - & 1 \\
\hline Hyd & - & 1 & - & - & - & - & - & - & - & $\therefore-$ & - & - & 1 \\
\hline Hydro & 218 & 229 & 124 & 78 & 66 & 69 & 33 & 144 & 105 ; & 81 & 67 & 35 & 1249 \\
\hline Hydro & 2 & 22 & $-{ }_{-}$ & - & 4 & 3 & 2 & - & 1 & - & - & - & 41 \\
\hline Lepto & 1 & - & 2 & - & - & - & - & - & $\therefore$ & - & - & - & 3 \\
\hline Philop & 281 & 18 & 6 & 14 & 14 & 18 & 23 & 122 & 220 & 102 & 64 & 1 & 883 \\
\hline Xiphoc & - & 1 & - & - & - & - & - & - & - & - & - & - & ${ }^{2}$ \\
\hline Total & 2755 & 1726 & 981 & 829 & 353 & 180 & 132 & 388 & 490 & 397 & 327 & 224 & 8782 \\
\hline $\mathrm{H}^{\prime}$ & 3,170 & 3,585 & 3,322 & 2,585 & 2,807 & 2,585 & 2,807 & 2,585 & 2,807 & 2,807 & 2,585 & 2,585 & 1,850 \\
\hline$E$ & 0,555 & 0,454 & 0,430 & 0,394 & 0,493 & 0,740 & 0,646 & 0,751 & 0,663 & 0,784 & 0,840 & 0,497 & 0,486 \\
\hline
\end{tabular}


Tabela II. Continuação

\begin{tabular}{|c|c|c|c|c|c|c|c|c|c|c|c|c|c|}
\hline & Ago/94 & Set & Out & Nov & Dez & Jan/95 & Fev & Mar & $\mathrm{Abr}$ & Mai & Jun & Jul & Total \\
\hline \multicolumn{14}{|l|}{ Ponto 4} \\
\hline Baetidae & 266 & 237 & - & 142 & 34 & 1 & 7 & 19 & 6 & 12 & 148 & 527 & 1399 \\
\hline Leptohyphidae & 427 & 126 & - & 329 & 109 & 10 & 20 & 93 & 48 & 71 & 257 & 271 & 1761 \\
\hline Leptophlebiidae & 195 & 125 & 1 & 183 & - & - & 6 & 9 & 14 & 12 & 16 & 1657 & 2218 \\
\hline Perlidae & 13 & 10 & - & 3 & - & - & - & $\therefore$ & - & 1 & 1 & 5 & 33 \\
\hline Calamoceratidae & - & 1 & - & - & 1 & - & - & - & - & - & - & - & 2 \\
\hline Glossosomatidae & 2 & 2 & - & 6 & - & - & - & - & - & - & - & 2 & 12 \\
\hline Helicopsychidae & 1 & - & 1 & 3 & - & - & - & - & - & - & - & 5 & 10 \\
\hline Hydrobiosidae & 1 & 3 & - & - & - & - & - & - & - & - & - & 1 & 5 \\
\hline Hydropsychidae & 147 & 306 & - & 370 & 53 & 17 & 42 & 18 & 33 & 37 & 101 & 68 & 1192 \\
\hline Hydroptilidae & 2 & 2 & - & 2 & 1 & 2 & 2 & - & - & - & - & - & 11 \\
\hline Leptoceridae & - & - & - & 1 & - & - & - & - & - & - & - & - & 1 \\
\hline Odontoceridae & - & 2 & - & - & - & - & - & 1 & - & - & - & - & 3 \\
\hline Philopotamidae & 14 & 60 & - & 95 & - & - & 1 & - & - & 1 & 5 & 10 & 186 \\
\hline Xiphocentronidae & 1 & 3 & - & - & - & - & - & - & - & - & - & - & 4 \\
\hline Total & 1069 & 877 & 2 & 1134 & 198 & 30 & 78 & 140 & 101 & 134 & 528 & 920 & 6837 \\
\hline $\mathrm{H}^{\prime}$ & 3,459 & 3,585 & 1,000 & 3,322 & 2,322 & 2,000 & 2,585 & 2,322 & 2,000 & 2,585 & 2,585 & 3,170 & 2,186 \\
\hline$E$ & 0,604 & 0,644 & 1,000 & 0,379 & 0,645 & 0,708 & 0,695 & 0,633 & 0,837 & 0,668 & 0,703 & 0,506 & 0,574 \\
\hline
\end{tabular}

Na coleta de outubro, ocorreu uma grande chuva que interferiu muito na comunidade, principalmente no ponto 4 . Este ponto é de quarta ordem, recebendo vários tributários. Dessa forma, a grande vazão registrada reflete o dia chuvoso e não a situação mensal, como já foi discutido. Nesse mês foram coletados somente dois indivíduos, indicando a nítida interferência da chuva na abundância. Nos demais pontos essa influência não foi tão drástica, já que os mesmos são de primeira e segunda ordens, não sofrendo o efeito acumulativo da água de outros afluentes, como acontece no Ribeirão João Leite (ponto 4).

A diversidade biológica é o resultado de eventos históricos e ecológicos, tanto regionais quanto locais, sendo geralmente diminuída pela desestabilização do sistema ecológico causado pela interferência antrópica. No Parque Ecológico de Goiânia a diversidade foi maior nos pontos $1 \mathrm{e} 4$. A nascente do ponto 1 é alterada pela criação de animais e desmatamento, porém o seu trecho dentro do parque é bem preservado, ocorrendo a formação de vários microhabitats devido as características geomorfológicas,. onde pode se estruturar vários nichos em uma pequena área. $\mathrm{O}$ trecho do ponto 4 apresentou um valor de diversidade esperado, principalmente em se tratando de um córrego de ordem média (quarta ordem), corroborando VANNOTE et al. (1980). Neste ponto a poluição orgânica, aparentemente, não foi limitante para a maioria das famílias de EPT, provavelmente pela diluição e autodepuração dos efluentes orgânicos. Os dois pontos de coleta no Córrego Carapina, apresentaram os menores valores do índice de diversidade, indicando a influência dos rebanhos bovinos que revolvem de maneira abrupta o substrato à jusante do trecho amostrado.

HYNES (1970), discutiu o papel da temperatura como fator fïsico importante em ambientes lóticos, o qual regula o ciclo de vida dos insetos aquáticos. A temperatura pode dificultar a dissolução de gases como o oxigênio na água (MAIER 1978), o que pode limitar a distribuição dos insetos aquáticos. No presente trabalho, a temperatura variou entre 14 e $23^{\circ} \mathrm{C}$, não se apresentando como um fator limitante para os grupos estudados. 
O oxigênio dissolvido no ambiente aquático é proveniente do gás atmosférico, bem como de organismos fotossintéticos (LIND 1979). Segundo VANNOTE et al. (1980), a atividade fotossintética pode ser reduzida com a turbidez da água, diminuindo a concentração de oxigênio. KLEEREKOPER (1944 apud MAIER 1978) considera que nos rios a difusão desse gás é aumentada pela turbulência das correntezas. Dessa forma, córregos de baixa ordem, que geralmente são encachoeirados, não possuem o oxigênio dissolvido em níveis limitantes.

Segundo MAIER (1978), geralmente os ambientes lóticos brasileiros, apresentam $\mathrm{pH}$ próximo da neutralidade, o que foi verificado no presente trabalho, onde variou de 6,8 a 7,9, com exceção do ponto 2, no mês de março, onde o pH apresentou se básico $(\mathrm{pH}=11) . \mathrm{O} \mathrm{pH}$, como no trabalho de FERREIRA \& FrOEHLICH (1992), não influenciou a fauna de EPT.

A condutividade elétrica é usada na determinação do teor de sais dissolvidos na água (material ionizado) (MAIER 1978; EsTEVES 1988). SCHROEDER-ARAúJO et al. (1986) constatou níveis baixos de condutividade elétrica em córregos do Parque Estadual de Campos do Jordão (São Paulo), os quais não ultrapassaram $40 \mu \mathrm{S} / \mathrm{cm}$. No caso do Parque Ecológico de Goiânia a condutividade ficou entre 60 e $150 \mu \mathrm{S} / \mathrm{cm}$. A condutividade não influenciou a abundância de EPT.

Na composição faunística brasileira são encontradas nove famílias de Ephemeroptera (DOMINGUEZ et al. 1992), duas de Plecoptera (FroEHLICH 1984b, 1990a) e 14 famílias de Trichoptera (R.R. Guahyba comunicacão pessoal). No do presente trabalho, foram coletadas quatro famílias de Ephemeroptera, uma de Plecoptera e onze de Trichoptera, sendo representados por $75 \%, 1 \%$ e $24 \%$ da abundância, respectivamente.

A comunidade dos EPT está estruturada de forma a aproveitar da melhor maneira possível os recursos ambientais (BISPO \& OLIVEIRA no prelo). A abundância desses organismos modifica-se no decorrer do ano, em resposta à variação de fatores ambientais ou simplesmente devido ao ciclo de vida (HYNES 1970). No presente trabalho, verificou-se que as maiores mudanças na abundância durante $o$ ano foi devido ao regime anual de chuvas e a conseqüente variação da vazão e da velocidade de correnteza da água.

AGRADECIMENTOS. Agradecemos a FUNAPE-UFG pelo apoio financeiro durante a realização do presente trabalho.

\section{REFERÊNCIAS BIBLIOGRÁFICAS}

AgRisANo, E.B. 1995. Insecta Trichoptera, p.1199-1237. In: E.C. LOPRETTO \& G. Tell (Eds). Ecosistemas de Aguas Continentales. Metodologias para su estudio. La Plata, Ediciones Sur, Vol. 3, 1401p.

BACHMANN, A.O. 1995. Insecta Plecoptera, p.1093-1111. In: E.C. LOPRETTO \& G.

Tell (Eds). Ecosistemas de Aguas Continentales. Metodologias para su estudio. Ediciones Sur, La Plata, Vol. 3, 1401 p.

BISPO, P.C. \& L.G. OliveirA. (no prelo). Distribuição espacial de insetos aquáticos 
(Ephemeroptera, Plecoptera e Trichoptera), em córregos de cerrado do Parque Ecológico de Goiânia, Estado de Goiás. In: L.N. Nessimian (Ed.) Oecologia Brasiliensis.

Boon, P.J.; B.P. JUPP \& D.G. LEE. 1986. The benthic ecology of rivers in the Blue Mountains (Jamaica) prior to construction of water regulation scheme. Arch. Hidrobiol./Suppl. 74: 315-355.

DOMINGUEZ, E.; M.D HubBard \& W.L. PeTERS. 1992. Clave para ninfas y adultos de las familias y generos de Ephemeroptera (Insecta) sudamericanos. Biologia Acuática. 16. La Plata, NLP-CONICET, 32p.

EsteVES, F.A. 1988. Fundamentos de Limnologia. Rio de Janeiro, Editora Interciência/FINEP, XI+575p.

Ferreira, M.J.N. \& C.G. Froehlich. 1992. Estudo da fauna de Ephemeroptera (Insecta) do Córrego do Pedregulho (Pedregulho, SP, Brasil) com aspectos da biologia de Thraulodes schlingeri Traver \& Edmunds, 1967. Revta bras. Ent. 36 (3): 541-548.

Froehlich, C.G. 1984a. Brazilian Plecoptera 3. Macrogynoplax veneranda sp. n. (Perlidae: Acroneuriinae). An. Limnol. 20 (1-2): 39-42.

1984b. Brazilian Plecoptera 4. Nymphs of perlid genera from southeastern Brazil. An. Limnol. 20 (1-2): 43-48.

1988. Brazilian Plecoptera 5. Old and new species of Kempnyia (Perlidae). Aquatic Insects 10 (3): 153-170.

. 1990a. Brazilian Plecoptera 6. Gripopteryx from Campos do Jordão, State of São Paulo (Gripopterygidae). St. Neotrop. Fauna Environ. 25 (4): 235-247. 1990b. Size variation in Kempnyia (Plecoptera:Perlidae), p.347-350. In:

I.C. CAMPBELl (Ed.). Mayflies and Stoneflies: Life Histories and Biology. Dordrecth, Kluwer Academic Publishers, X+366p.

1991. Flight periods of Kempnyia and Macrogynoplax (Plecoptera: Perlidae) in southeastern Brazil, p.353-357. In: J. AlBA-TERCEDOR \& A. SANCHEZ-ORTEGA (Eds). Overview and Strategies of Ephemeroptera and Plecoptera. Gainesville, The Sandhill Crane Press, Inc., XIV+588p.

1994. Brazilian Plecoptera 8. On Paragripopteryx (Gripopterygidae). Aquatic Insects 16 (3): 1-13.

1996. Two new species of Kempnyia from southern Brazil (Plecoptera:

Perlidae). Bull. Soc. Ent. Suisse 69: 117-120.

GuAHYBA, R.R. 1988. Contribuição ao estudo das formas imaturas da ordem Trichoptera Kirby, 1813, da Represa dos Ciganos, RJ (Insecta). Acta. Limnol. Brasil. 2: 751-769.

HyNES, H.B.N. 1970. The Ecology of Running Waters. Ontario, Liverpool University Press, 555p.

LIND, O.T. 1979. Handbook of Common Methods in Limnology. London, The C.V. Mosby Company, 199p.

MAIER, M.H. 1978. Considerações sobre características limnológicas de ambientes lóticos. Bolm. Inst. Pesca 5 (2): 75-90.

MagurRan, A.E. 1991. Ecological Diversity and It's Measurement. London, 
Chapman \& Hall, 178p.

MERRITT, R.W. \& K.W. CUMmins. 1979. An Introduction to the Aquatic Insects of North America. Iowa, Ed. Kendall/Hunt Publishing Company, $2^{\text {nd }}$ ed., $441 \mathrm{p}$. NIMER, E. 1989. Climatologia do Brasil. Rio de Janeiro, IBGE, $421 \mathrm{p}$.

Peterson, R.H. \& L.V. EecKhaute. 1992. Distributions of Ephemeroptera, Plecoptera, and Trichoptera of three maritime catchments differing in $\mathrm{pH}$. Freshwater Biology 27: 65-78.

Oliveira, L.G. \& C.G. FroeHLich. 1996. Natural history of three Hydropsychidae (Trichoptera:Insecta) in a "cerrado" stream from northeastern São Paulo State, Brazil. Revta bras. Zool. 13 (3): 755-762.

Schroeder-Araujo, L.T.; H.L. STEMPNiEWISKI; M.N. Cipolli; L.E. SANTOS; M. SANTO-PAUlo \& W. CORRÊA-CrEMONESI. 1986. Estudo limnológico e climático da região do Parque Estadual de Campos do Jordão, SP, com vistas ao povoamento com truta arco-íris, Salmo irideus Gibbons. Bolm. Inst. Pesca 12 (3): 63-76.

SChroeder-Araujo, L.T. \& M.N. Cipolli. 1986. Organismos bentônicos como indicadores da qualidade de água de rios no Parque Estadual de Campos do Jordão, SP. Bolm. Inst. Pesca 12 (3): 77-83.

SLOBODCHIKofF, C.N. \& J.E. PARROTT. 1977. Seazonal diversity in aquatic insect communities in an all-year stream system. Hydrobiologia 52 (2-3): 143-151.

STRAHLER, H.N. 1957. Quantitative analysis of watershed geomorphology. Amer. Geophys. Union Trans. 33: 913-920.

VAnNote, R.L.; G.W Minshall; K.W.L Cummins; J.R. SEDELL \& C.E Cushing. 1980. The River Continuum Concept. Can. Jour. Fish. Aquat. Sci. 37: 130137.

Vanzolini, P.E. 1964. História Natural de Organismos Aquáticos do Brasil. FAPESP, São Paulo, 452p.

WARD, J.V. \& J.A. STANFORD. 1982. Thermal responses in the evolutionary ecology of aquatic insects. Ann. Rev. Entomol. 27: 97-117.

Zamora-Muñoz, C.; A. Sanchez-Ortega \& J. Alba-Tercedor. 1993. Physico-chemical factors that determine the distribution of mayflies and stoneflies in a high-mountain stream in Southern Europe (Sierra Nevada, Southern Spain). Aquatic Insects 15 (1): 11-20.

Recebido em 28.II.1997; aceito em 22.X.1997. 